\title{
Cooperative Diversity with Disconnection Constraints and Sleep Discipline for Power Control in Wireless Sensor Networks
}

\author{
C. Fischione, A. Bonivento, K. H. Johansson, A. Sangiovanni-Vincentelli
}

\begin{abstract}
We derive a power control policy for a group of sensor nodes that are monitoring a real-time application sensitive to disconnections (outages) of the communication. Specifically, we suggest that the sensor nodes perform cooperative diversity while running a sleep discipline. After the description of a detailed model of the wireless links, we propose a power minimization algorithm with a constraint expressed in terms of outage probability. Suboptimal solutions are also discussed. Numerical examples are provided for various number of nodes, wireless scenarios and nodes activities. It is argued that nodes with reduced activity show better performance.
\end{abstract}

Index Terms: Wireless Sensor Networks (WSNs), Cooperative Diversity, Fading, Outage.

\section{INTRODUCTION}

A major challenge in the design of systems based on wireless sensor networks (WSNs) is to achieve reliable performance out of unreliable components. A general way to tackle this problem is to utilize the node densities and group properties. Cooperative diversity is a technique that exploits groups of sensor nodes randomly placed to cooperatively relay a commonly received signal toward a destination with the goal to combat severe attenuations or disconnections of the signal strength.

In recent years, cooperative diversity has received attention for cellular radio systems and ad-hoc wireless networks. Such systems, however, are usually equipped with high processing capability. On the contrary, the nodes of a WSN have limited memory and power resources and are usually deployed in unfriendly environment, where recharging and maintenance is not possible. To ensure an adequate lifetime, WSN protocols often leverage cross-layer design techniques to minimize energy consumption [1], [2]. Furthermore, sensor nodes are often part of WSNs where several complementary techniques and protocols are simultaneously running, as e.g. collaborative localization [3], distributed source coding [4], and sleep discipline [5], [6]. Consequently, an effective cooperative diversity protocol for WSNs must be able to operate together with these

C. Fischione and K.H. Johansson are with the Automatic Control Lab, School of Electrical Engineering, KTH, SE 100 44, Stockholm, Sweden, $\{$ carlofilkallej\}@ee.kth.se; A. Bonivento and A. Sangiovanni-Vincentelli are with the EECS Dept., University of California at Berkeley, Berkeley, CA 94 720, $\{$ alviselalberto\} deecs. berkeley. edu.

Work done in the framework of the HYCON Network of Exellence, contract number FP6-IST-511368. The work by C. Fischione and K. H. Johansson was partially funded also by the Swedish Foundation for Strategic Research through an Individual Grant for the Advancement of Research Leaders and by the Swedish Research Council. cross-layer techniques and contribute to minimizing energy consumption.

In this paper, we investigate the problem of power control of nodes performing cooperative diversity with sleep discipline and outage constraints. Specifically, we minimize the power consumption while guaranteeing a maximum outage probability of the signal. We resort to an accurate framework for the abstraction of the salient properties of Signal to Interference + Noise Ratio (SINR), including all the relevant parameters that may influence the upper layer protocols and applications, and extend the outage analysis proposed in [7] to include the activity status of the sensor nodes.

Disconnection events have strong influence on the performance of upper layers in the protocol stack, e.g., [8], [9], [7]. An example is when a WSN is used to gather information for real-time control of a plant sensible to the delays of the communication. The stability of the closed-loop control system may require rates of signal disconnections lower than a certain frequency related to the sampling frequency of the sensors and actuators as well as the dynamics of the plant. The importance of outage events for WSNs was also recognized in [10], where the authors characterize the wireless links for WSNs as connected, transitional and disconnected.

Some recent contributions can be found in the literature about cooperative diversity for ad-hoc and WSNs. In [11], the authors propose schemes for cooperation diversity for pairs of users in cellular networks, but they recognize the importance of this technique also for ad-hoc networks. In [12] the authors investigate the possibility of shaping the radiation diagram using the random distribution of nodes within a WSN. However, the analysis is based on a number of ideal assumptions, particularly a simple model of the wireless channel. In [13] and [14], an interesting framework is addressed, where nodes are grouped in clusters and collaborate to receive and transmit information using a distributed maximalratio combining algorithm. In [15] and [16], several schemes of cooperative diversity are investigated, including relay and forward, decode and forward, and schemes with space-time coding.

Our approach differs from [13] and [14], because we focus on the power minimization under outage probability constraint instead of optimally shaping the equivalent radiation diagram of the sensor array. For real-time applications, the outage probability is a more reasonable measure than the bit error probability analysis for delay-tolerant applications addressed in [16]. We present a realistic link layer model, which include path-loss, slow fading and Rayleigh fading while [15] - [17] 
include only Rayleigh fading. Furthermore, the analytical tool therein developed for characterizing the outage probability are valid only for high Signal to Noise Ratio, while our study is more general.

Finally, none of the contributions that can be found in the literature take into account the presence of sleep disciplines. Different sleeping protocols have been proposed. In [6], [18], [19], nodes periodically turn off their radio according to a predetermined schedule that allows for maintaining network connectivity. In [5], nodes go to sleep for a randomized amount of time, while end-to-end performance is maintained relying on node densities. In this paper, we model the node activity abstracting the details of the sleeping protocol and show how the outage probability depends only on the average duty-cycle. Consequently, the proposed cooperative diversity technique can operate on top of any of the sleeping disciplines.

The rest of the paper is organized as follows: in Section II, we describe the system scenario; in Section III, the wireless channel is accurately described; in Section IV, the power optimization problem is described and discussed; in Section $\mathrm{IV}$, the outage probability is derived; in Section V numerical examples are discussed, and in Section VI conclusions and future developments are given.

\section{SySTEM SCENARIO}

Consider the scenario in Fig. 1. We have a moving sensor node, called MS, that broadcasts a signal associated to a real time application. There is a cluster of $N$ sensor nodes that receive the broadcasted signal and retransmit it to a Sensor Collector (SC) that either has higher computation capabilities, or is directly connected to such a computation resource. For example, the MS could transmit the status information associated to an inverted pendulum as in [1], where the communication between the actuator at the MS and the controller at the SC employs a WSN that introduces constraints on the loop stability.

There is no direct link between the MS and the SC, the signal has to pass through the relaying nodes that cooperatively weight the received signals to improve the quality of the signal eventually received by the $\mathrm{SC}$. The sensor nodes transmit using different channels, for example they could use two different frequencies, time slots or codes. We assume that the retransmission of signals happens with no appreciable delay.

The links between the MS and the relaying nodes are frequency flat, slowly time variant and affected by path loss, shadow and fast fading. This assumptions are representative of the Industrial, Scientific, and Medical (ISM) transceivers, which are commonly employed in WSNs [20], [21]. We assume the relay sensor nodes and the SC fixed and in visibility. Consequently, the channel coefficients describing the links between them are assumed known with a good approximation. This set up is basically equivalent to the one considered in [13].

\section{Channel Model}

The channel gain between the moving sensor node and the generic node $i$ is denoted with $h_{i}(t)$, and is comprehensive

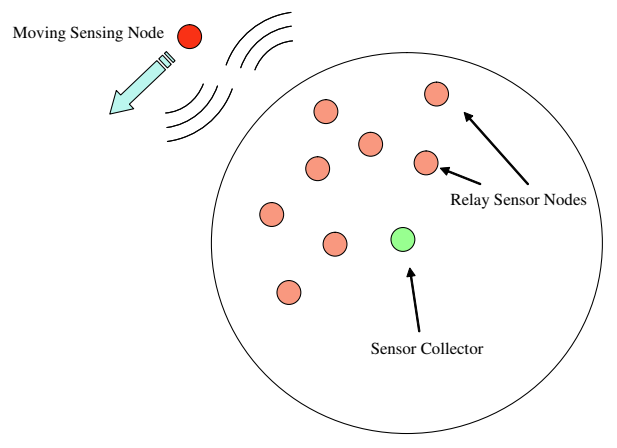

Fig. 1. System overview of the Wireless Sensor Network.

of path-loss, shadowing and fast fading. We express such a coefficient using a multiplicative model [22]:

$$
h_{i}(t)=g_{i}(t) \sqrt{L_{i}(t) P_{L}\left(d_{i}\right)},
$$

where

$$
g_{i}(t)=\left|g_{i}(t)\right| e^{j \phi_{i}(t)}
$$

is the fast fading, $L_{i}(t)$ is the shadowing, and $P_{L}\left(d_{i}\right)$ is the path-loss attenuation computed at $d_{i}$, the distance from the MS and the receiver $i$. A common assumption for $g_{i}(t)$ is to be a complex zero mean Gaussian random process, then $\left|g_{i}(t)\right|$ is Rayleigh distributed. Moreover, let us define

$$
p_{i}(t) \triangleq\left|g_{i}(t)\right|^{2},
$$

then, $p_{i}(t)$ is an exponentially distributed process, with parameter $\sigma_{p_{i}}$. The average and correlation of $p_{i}(t)$ are given as in the following [22]:

$$
\begin{gathered}
E\left\{p_{i}(t)\right\} \triangleq m_{p_{i}}=2 \sigma_{p_{i}}^{2}, \\
E\left\{p_{i}^{2}(t)\right\} \triangleq r_{p_{i}}=8 \sigma_{p_{i}}^{4},
\end{gathered}
$$

Furthermore, $g_{i}(t)$ and $g_{j}(t)$, with $i \neq j$, are statistically independent.

The shadowing component $L_{i}(t)$ is characterized with a lognormal distribution

$$
L_{i}(t)=e^{B_{i}(t)}
$$

where $B_{i}(t)$ is a Gaussian random process having average $m_{B_{i}}$ and variance $\sigma_{B_{i}}^{2}$. The shadowing component exhibits a correlation structure that is dependent on the physical properties of the propagation scenario. Since performances of spatial diversity schemes are strongly influenced by correlation properties, it is fundamental the adoption of realistic models. Consequently, we include in the model the cross-correlation function derived in [23]:

$$
C_{B_{i} B_{j}}=\sigma_{B_{i}} \sigma_{B_{j}} A \cos \left[\vartheta_{i j}(t)+B\right],
$$

where $\vartheta_{i j}(t)$ is the angle between the links from the MS and the node $i$, and the MS and the node $j$, and $A$ and $B$ are two constants such that $A+B \leq 1$. The values for $A$ and $B$ are dependent on the propagation environment. The average and autocorrelation function of $L_{i}(t)$ can be expressed as follows:

$$
\begin{gathered}
E\left\{L_{i}(t)\right\}=e^{m_{B_{i}}+\frac{1}{2} \sigma_{B_{i}}^{2}}, \\
E\left\{L_{i}(t) L_{j}(t)\right\}=e^{m_{B_{i}}+m_{B_{j}}+\frac{1}{2}\left[\sigma_{B_{i}}^{2}+\sigma_{B_{j}}^{2}+2 C_{B_{i} B_{j}}\right] .}
\end{gathered}
$$


The path-loss term is expressed, in $\mathrm{dB}$, as follows [10]:

$$
P_{L}(d)_{d B}=-P_{L}\left(d_{r}\right)_{d B}-10 \ell \log _{10}\left(\frac{d_{i}}{d_{r}}\right),
$$

where $P_{L}\left(d_{r}\right)_{d B}$ is the path loss computed at the reference distance $d_{r} ; \ell$ is the path-loss decay constant, and lays in the interval 2..4;

The generic node $i$ is not continuously awake, but may be in a sleep condition. The activity state of the node, at a given time $\mathrm{t}$, is modelled with the binary random variable $\nu_{i}(t)$, with $P\left[\nu_{i}(t)=1\right]=\mu_{i}$ and $P\left[\nu_{i}(t)=0\right]=1-\mu_{i}$, where $\mu_{i}$ is said to be the activity factor of the node. Moreover, the node $i$, after weighting the received signal $s(t)$ at the coherent FSK receiver with the complex coefficient $e^{-j \phi_{i}(t)}$, retransmits it with an appropriate power level $P_{i}$ toward the SC. This procedures are compatible with on-the-shelf products as e.g. Tmote [21].

The SC receives simultaneously the retransmitted signals from the various sensor nodes, and therefore performs in automatic way the sum of the signals. The complex envelope of the received signal is, hence, expressed as follows:

$$
y(t)=\sum_{i=1}^{N} w_{i} \nu_{i}(t)\left[h_{i}(t) s(t)+n_{i}(t)\right]+n(t)
$$

where $w_{i}=\sqrt{P_{i}} e^{-j \phi_{i}(t)}, i=1, \ldots, N$ are the complex values of the node weighting coefficients, and for the sake of notation simplicity, we include in the node power $P_{i}$ also the known channel gains between the nodes and the SC; $n_{i}(t)$ is the noise component at the node $i$, and is assumed as white Gaussian noise (AWGN), with power spectral density given by $\sigma_{i}^{2}$; finally, $n(t)$ includes the interferences and AWGN noise present at the receiver of the SC, having power spectral density $\sigma_{n}^{2}$.

We denote the power coefficient with the vector $\mathbf{P}=$ $\left[P_{1}, P_{2}, \ldots, P_{N}\right]$, the fading coefficients with the vector $\boldsymbol{h}=$ $\left[h_{1}(t), h_{2}(t), \ldots, h_{N}(t)\right]$ and the activity factors with the vector $\boldsymbol{\nu}=\left[\nu_{1}(t), \nu_{2}(t), \ldots, \nu_{N}(t)\right]$.

We express the Signal to Interference + Noise Ratio (SINR) corresponding to (11) as follows [22]:

$$
\operatorname{SINR}(\boldsymbol{P}, \boldsymbol{h}, \boldsymbol{\nu})=\frac{E_{s} \sum_{i=1}^{N} P_{i} \nu_{i}(t) r_{i}(t)}{\sum_{i=1}^{N} P_{i} \nu_{i}(t) \sigma_{i}+\sigma_{n}},
$$

where $E_{s}$ is the energy of the transmitted signal, while $r_{i}(t)=$ $\left|h_{i}(t)\right|^{2}$. Note that dependence of the SINR on the powers of the relaying nodes, the channel gains and the activity factors has been evidenced. Moreover, note that (12) is a time variant stochastic process since the fading is assumed to be time variant.

The signal (11) is said to be in outage or disconnected if the SINR (12) falls below a minimum quality threshold $\gamma$. In particular, the probability of the outage events is defined as follows:

$$
P_{\text {out }}=P[\operatorname{SINR}(\boldsymbol{P}, \boldsymbol{h}, \boldsymbol{\nu})<\gamma] .
$$

\section{Outage-Based Power Minimization Algorithm}

Call $\bar{P}_{\text {out }}$ the maximum allowed value of the outage probability. The minimization of the transmission powers of the sensors, under the constraint on the outage probability, becomes:

$$
\left\{\begin{array}{cl}
\min _{\mathbf{P}} & \sum_{i=1}^{N} \rho_{i} \mu_{i} P_{i} \\
\text { s.t. } & P[S I N R(\boldsymbol{P}, \boldsymbol{h}, \boldsymbol{\nu})<\gamma]<\bar{P}_{\text {out }} \\
& P_{\text {min }} \leq P_{i} \leq P_{\text {max }} i=1, \ldots, N
\end{array}\right.
$$

where $P_{\min }$ and $P_{\max }$ are the minimum and maximum values allowed for the power coefficient; $\rho_{i} \in[0,1] i=$ $1, \ldots, N$ is an adaptive constant that represents the residual energy of the relay nodes.

Since the outage constraint is a non-linear function with a complex dependence on power coefficients, the solution of the optimization problem is derived at the SC with a numerical algorithm. This is not a difficult task, since we assume that the $\mathrm{SC}$ is directly connected with high computational resources. However, our future work will be focused on finding closedform approximations of the optimal solution.

The solution of the optimization problem requires the estimation of the fading parameters by the nodes, that subsequently retransmit them to the SC. Note that it is not required the deterministic knowledge of the fading coefficients for the computation of the outage probability, but, as it will be clear in section $\mathrm{V}$, only the estimation of the first and second order moments. Such estimations can be easily performed by each node by e.g. running average or recursive low-pass filters. Consequently, the cost of the estimation operations is computationally reduced and, hence, suitable for sensor nodes. Moreover, since the channel parameters vary with a frequency corresponding to the coherence time of the wireless propagation, the parameters estimations, and thus the powers, should be updated at least with the same time scale, which is of the order of hundreds of $m s$ (according to the MS mobility).

Once the powers are optimized at the SC, they have to be transmitted to the nodes. This transmission may be expensive in terms of energy consumption, delay, etc. especially when the number of nodes involved is high. To avoid such an onerous retransmission, distributed and suboptimal solutions could be investigated. For example, each node $i$ may have stored a set of $M$ powers coefficients $P_{i, j}, j=1, \ldots, M$, pre-computed according to a set of $M$ channel conditions and outage constraints. Each possible combination of powers among nodes could be associated to an index. Therefore, the SC could transmit to the nodes just the index corresponding to the combination, thus saving considerable amount of resources.

\section{Derivation of the Outage Probability}

The computation of the outage probability constraint in (14) requires the characterization of the statistics of the SINR (12). However, the SINR is a non linear function of several stochastic processes: the log-normal fading and the Rayleigh fading coefficients, and the activity processes. We approach the problem of the derivation of the outage probability by following two steps: first we assume that the activity factors are fixed and compute the outage probability conditioned on the activity status. Subsequently, the conditional outage probability is averaged with respect to the activity status distributions. 
Given one of the $2^{N}$ possible realization of the activity vector $\boldsymbol{\nu}$, the SINR is expressed as a linear combination of Reyleigh-Lognormal random processes, and we can rely upon the extension of the Wilkinson Moment matching method [24] [25]. Specifically, we consider the following approximation:

$$
S I N R(\boldsymbol{P}, \boldsymbol{h}, \boldsymbol{\nu}) \cong e^{Z(\boldsymbol{P}, \boldsymbol{h}, \boldsymbol{\nu})},
$$

where $Z(\boldsymbol{P}, \boldsymbol{h}, \boldsymbol{\nu})$ is a Gaussian random process having average $m_{Z}(\boldsymbol{P}, \boldsymbol{\nu})$ and covariance $\sigma_{Z}(\boldsymbol{P}, \boldsymbol{\nu})$, that are derived as a function of the first and second moments of the SINR:

$$
\begin{gathered}
m_{Z}(\boldsymbol{P}, \boldsymbol{\nu})=\ln \left[\frac{M_{m 1}^{2}(\boldsymbol{P}, \boldsymbol{\nu})}{\sqrt{M_{m 2}(\boldsymbol{P}, \boldsymbol{\nu})}}\right], \\
\sigma_{Z}^{2}(\boldsymbol{P}, \boldsymbol{\nu})=\ln \left[\frac{M_{m 2}(\boldsymbol{P}, \boldsymbol{\nu})}{M_{m 1}^{2}(\boldsymbol{P}, \boldsymbol{\nu})}\right],
\end{gathered}
$$

where:

$$
\begin{aligned}
& M_{m 1}(\boldsymbol{P}, \boldsymbol{\nu}) \triangleq E_{\boldsymbol{h}}\{S I N R(\boldsymbol{P}, \boldsymbol{h}, \boldsymbol{\nu}) \mid \boldsymbol{\nu}\} \\
& M_{m 2}(\boldsymbol{P}, \boldsymbol{\nu}) \triangleq E_{\boldsymbol{h}}\left\{S I N R^{2}(\boldsymbol{P}, \boldsymbol{h}, \boldsymbol{\nu}) \mid \boldsymbol{\nu}\right\} .
\end{aligned}
$$

In the Appendix, the expressions of (18) and (19) are provided.

The SINR in $d B, S I N R_{d B}$, is a Gaussian process, and is expressed as follows:

$$
S I N R_{d B}(\boldsymbol{P}, \boldsymbol{h}, \boldsymbol{\nu})=\beta Z(\boldsymbol{P}, \boldsymbol{h}, \boldsymbol{\nu}),
$$

with average and covariance function expressed, respectively, as $m_{S I N R_{d B}}(\boldsymbol{P}, \boldsymbol{\nu})=\beta m_{Z}(\boldsymbol{P}, \boldsymbol{\nu})$ and $\sigma_{S I N R_{d B}}(\boldsymbol{P}, \boldsymbol{\nu})=$ $\beta^{2} \sigma_{Z}(\boldsymbol{P}, \boldsymbol{\nu})$, with $\beta=10 / \ln 10$.

The $S I N R_{d B}$ Gaussian distribution allows for a straightforward derivation of outage probability conditioned on the distribution of $\nu$,

$$
P_{\text {out }}(\boldsymbol{P} \mid \boldsymbol{\nu})=Q(u)
$$

where

$$
Q(x)=\frac{1}{\sqrt{2 \pi}} \int_{x}^{\infty} e^{-\frac{y^{2}}{2}} d y
$$

and

$$
u=\frac{m_{S I N R_{d B}}(\boldsymbol{P}, \boldsymbol{\nu})-\gamma_{d B}}{\sigma_{S I N R_{d B}}(\boldsymbol{P}, \boldsymbol{\nu})}=\frac{\beta m_{Z}(\boldsymbol{P}, \boldsymbol{\nu})-\gamma_{d B}}{\beta \sigma_{Z}(\boldsymbol{P}, \boldsymbol{\nu})},
$$

and $\gamma_{d B}=10 \log \gamma$.

Finally, the outage probability (13) can be evaluated averaging with respect to the probability mass function of the vector $\boldsymbol{\nu}$, thus obtaining the following expression:

$$
\begin{aligned}
P[S I N R(\boldsymbol{P}, \boldsymbol{h}, \boldsymbol{\nu})<\gamma] & =E_{\boldsymbol{\nu}}\left\{P_{\text {out }}(\boldsymbol{P} \mid \boldsymbol{\nu})\right\} \\
& =\sum_{k=1}^{2^{N}} P_{\text {out }}\left(\boldsymbol{P} \mid \boldsymbol{\nu}_{\boldsymbol{k}}\right) P\left(\boldsymbol{\nu}_{\boldsymbol{k}}\right)
\end{aligned}
$$

where $P\left(\boldsymbol{\nu}_{\boldsymbol{k}}\right)$ is the probability of $\boldsymbol{\nu}_{\boldsymbol{k}}$. Specifically, call the realization $\boldsymbol{\nu}_{\boldsymbol{k}}=\left[\delta_{i, k}\right]$, with $i=1, \ldots, N$, and $\delta_{i, k}=1$ if the sensor $i$ is active, and $\delta_{i, k}=0$ is the sensor is sleeping. Hence, the probability of such a vector is expressed as

$$
P\left(\boldsymbol{\nu}_{\boldsymbol{k}}\right)=\prod_{i=1}^{N}\left[\delta_{i, k}\left(2 \mu_{i}-1\right)+1-\mu_{i}\right] .
$$

\section{NUMERICAL EXAMPLES}

In this section, numerical examples are derived and discussed. We consider the same scenario adopted in [10]. Specifically. we assume that the MS transmits with a power of $0 d B_{m}$, and $P_{L}\left(d_{r}\right)_{d B}=-55 d B$. The power spectral density of the noise for the relay nodes is assumed $n_{i}=-115 d B_{m}$, for $i=1, \ldots, N$, and the same value is set for the SC. The relay powers are assumed to lay in the interval $-30 d B, \ldots, 0 d B$. We further assume $\gamma=7.6 \mathrm{~dB}[10], \bar{P}_{\text {out }}=0.01$, and $\rho_{i}=1.0$.

We consider two wireless scenarios where the optimization problem (14) is solved for different numbers of nodes $(3,5$, and 8$)$ and different values of the node activity factors $(0.3$, 0.5 and 0.9 ). Without loss of generality, nodes are set to the same activity factor. In the first scenario, the relay nodes are randomly placed between 5 and 10 meters from the MS and are assumed to experience the same standard deviation of the shadowing $\sigma_{B_{i}}=4$ and the same standard deviation of the fast fading $\sigma_{p_{i}}=0.1$. In the second scenario, the relay nodes are randomly placed between 3 and 15 meters from the MS, with a standard deviation of the shadowing uniformly distributed between 2 and 4 , and with a standard deviation of the fast fading uniformly distributed between 0.1 and 0.4 . This means that the second scenario has shorter fluctuations of the shadowing components, but deeper fluctuations of the fast fading.

In Figure 2, the value of the optimized objective function is reported for the first scenario. As the number of nodes increases, the total power consumption increases in the cases $\mu_{i}=0.5$ and 0.9 , while the case 0.3 exhibits a slight decreasing. This case is the most relevant since the relay nodes experience a low duty cycle and, in the same time, are perfectly able to guarantee the performance set by the constraints.

In Figure 3 the numerical results of the second scenario are reported. As in the previous scenario, the curves confirms that better performance are obtained with lower values of the activity factor. However, as the number of nodes increases, there is a decrease of the total power consumption. This difference can be explained by observing that the fluctuations of the shadowing components are reduced with respect to the first scenario, and hence the outage events are shorter.

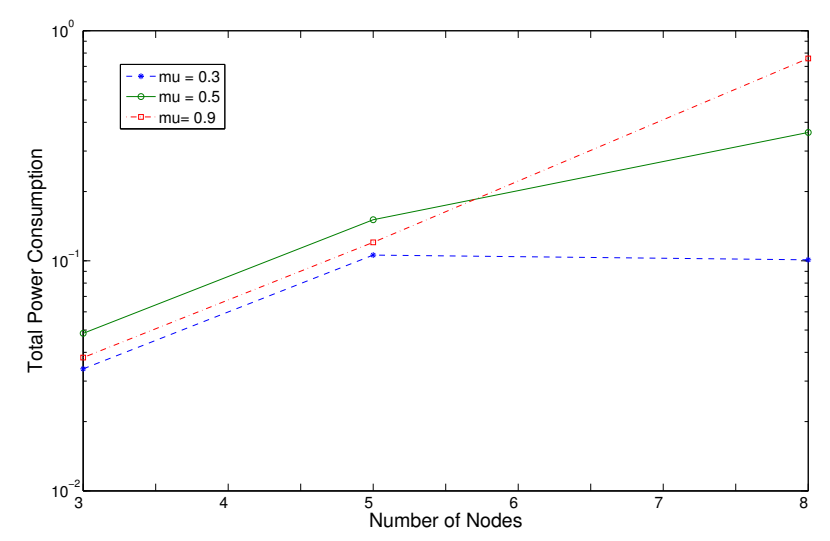

Fig. 2. Power consumption $(d B / 10)$ for the first scenario and three cases of the activity factor as function of the number of relay nodes. 


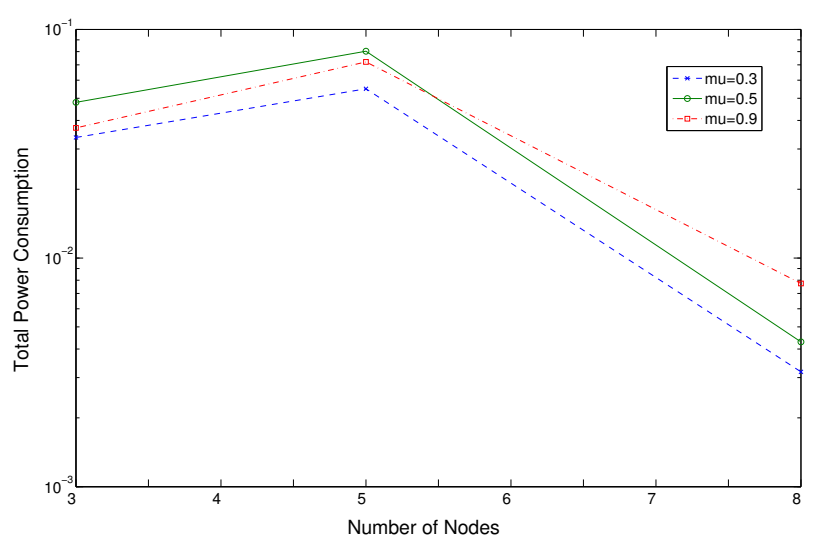

Fig. 3. Power consumption $(d B / 10)$ for the second scenario and three cases of the activity factor as function of the number of relay nodes.

\section{Conclusions And Future Developments}

We have proposed an optimization problem for the minimization of power consumption for a group of sensor nodes performing cooperative diversity and running sleep discipline under signal connectivity constraints. The optimization problem includes as constraint the outage probability of the received signal. Specifically, the outage probability was derived after approximating the SINR statistics with the extended Wilkinson moment matching method, and then averaging with respect to the sensor nodes activity distributions. Our methodology is general and can be applied together with most of the proposed sleeping disciplines.

Numerical results show that in the scenario we have considered the power consumption can be minimized while satisfying the constraints. Moreover, reduced node activities shows better performances. However, an investigation on a larger number of scenarios should be carried out in order to draw general conclusions.

We plan to extend our work for multi-hop sensor networks and to include in the optimization problem also the second order statistics (average duration) of the outages [7]. Furthermore, in this paper, the activity factor and the power coefficients are regarded independent, whereas the method we have proposed could be extended considering a statistical correlation among the residual powers and activity factors.

\section{APPENDIX}

The first and second order moments of (12) can be derived as follows:

$$
\begin{aligned}
M_{m 1}(\boldsymbol{P}, \boldsymbol{\nu})= & C A \sum_{i=1}^{N} \nu_{i}(t) P_{i} 2 \sigma_{p_{i}}^{2} e^{m_{B_{i}}+\frac{1}{2} \sigma_{B_{i}}^{2}} \\
M_{m 2}(\boldsymbol{P}, \boldsymbol{\nu})= & C A^{2} \sum_{i=1, i \neq j}^{N} \sum_{j=1}^{N} \nu_{i}(t) \nu_{j}(t) P_{i} P_{j} 2 \sigma_{p_{i}}^{2} 2 \sigma_{p_{j}}^{2} \\
& e^{m_{B_{i}}+m_{B_{j}}+\frac{1}{2}\left[\sigma_{B_{i}}^{2}+\sigma_{B_{j}}^{2}+2 C_{B_{i} B_{j}}\right]}+ \\
& C A^{2} \sum_{i=1}^{N} \nu_{i}(t) P_{i}^{2} \sigma_{p_{i}}^{8} e^{2 m_{B_{i}}+2 \sigma_{B_{i}}^{2}}
\end{aligned}
$$

where:

$$
C A=\frac{E_{s}}{\sum_{i=1}^{N} \nu_{i}(t) P_{i} \sigma_{i}+\sigma_{n}} .
$$

\section{REFERENCES}

[1] X. Liu, A. Goldsmith. Wireless network design for distributed control. IEEE CDC, 2004.

[2] M. Sgroi, A. Wolisz, A. Sangiovanni-Vincentelli, J. M. Rabaey. A service-based universal application interface for ad-hoc wireless sensor networks. whitepaper, U.C.Berkeley, 2004.

[3] A. Savvides, M.B. Srivastava. Distributed fine-grained localization in ad-hoc networks. IEEE Transactions of Mobile Computing, 2003.

[4] J. Chou, D. Petrovic, K. Ramchandran. A distributed and adaptive signal processing approach to reducing energy consumption in sensor networks. IEEE INFOCOM, 2003.

[5] J. van Greunen, D. Petrovic, A. Bonivento. Adaptive sleep discipline for energy conservation and robustness in dense sensor networks. In Proc. of IEEE ICC 2004, 2004.

[6] Wei Ye, John Heidemann and Deborah Estrin. Medium access control with coordinated adaptive sleeping for wireless sensor networks. IEEE/ACM Transactions on Networking, Vol. 12, No. 3, pp. 493-506, June 2004.

[7] C. Fischione, A. Bonivento, A. Sangiovanni-Vincentelli, F. Santucci, K. H. Johansson. Performance analysis of collaborative spatio-temporal processing for wireless sensor networks. In Proc. of IEEE CCNC, January 2006.

[8] M. Zorzi. Outage and error events in bursty channels. IEEE Transactions on Communications, Vol. 46, no.3, 1998.

[9] L. Yang, M. S. Alouini. On the average outage rate and average outage duration of wireless communication systems with multiple cochannel interferers. IEEE Transaction on Wireless Communications, Vol. 3, N. 4, July 2004.

[10] M. Zuniga, B. Krishnamachari. Analyzing the transitional region in low power wireless links. SECON, 2004.

[11] A. Sedonaris, E. Erkio, B. Aazhang. User cooperation diversity part i: System description; and user cooperation diversity - part ii: Implementation aspects and performance analysis. IEEE Trans. on Communications, Vol. 51, N. 11, November 2003.

[12] H. Ochiai, P. Mitran, H. V. Poor, V. Tarokh. Collaoborative beamforming in ad hoc networks. IEEE Information Theory Workshop, 2004.

[13] R. Mudumbai, J. Hespanha, U. Madhow, G. Barriac. Scalable feedback control for distributed beamforming in sensor networks. IEEE International Symposium on Information Theory, 2005.

[14] G. Barriac, R. Mudumbai, U. Madhow. Distributed beamforming for information transfer in sensor networks. IEEE IPSN04, 2004.

[15] L. N. Laneman, G. W. Wornell. Distributed space-time-coded protocols for exploiting cooperative diversity in wireless networks. IEEE Trans. on Inf. Theory, Vol. 49, N. 10, October 2003.

[16] D. Chen, J. N. Laneman. Cooperative diversity for wireless fading channels without channel state information. IEEE Asilomar Conference, 2004.

[17] J. N. Laneman, D. N. C. Tse, G. Wornell. Cooperative diversity in wireless networks: Efficient protocols and outage behavior. IEEE Trans. on Info. Tehory, Vol. 50, N. 12, December 2004.

[18] J. van Greunen, D. Petrovic, A. Bonivento. Geography-informed energy conservation for ad hoc routing. MobiCom 2001, pp. 70-84, July 2001.

[19] A. Bonivento, C. Fischione, A. Sangiovanni-Vincentelli, F. Graziosi, F. Santucci. Seran: A semi random protocol solution for clustered wireless sensor networks. In Proc. of IEEE MASS 05, October 2005.

[20] H. Karvonen, Z. Shelby, C. Pomalaza-Rez. Coding for energy efficient wireless embedded networks. IWWAN, 2004.

[21] J. Polastre, R. Szewczyk, D. Culler, "Telos: Enabling Ultra-Low Power Wireless Research. Telos: Enabling ultra-low power wireless research. IPSN/SPOTS, April, 2005.

[22] G. L. Stüber. Priciples od Mobile Communication. Kluwer Academic Publishers, 1996.

[23] F. Graziosi, F. Santucci. A general correlation model for shadow fading in mobile radio systems. IEEE Communication Letters, Vol. 6, No. 3, March 2002.

[24] F. Graziosi, F. Santucci. On sir fade statistics in rayleigh-lognormal channels. IEEE ICC 2002, Vol. 3, 2002.

[25] F. Santucci, G. Durastante, F. Graziosi, C. Fischione. Power allocation and control in multimedia cdma wireless systems. Kluwer Telecommunication Systems, Vol. 23, pp. 69-94, May-June 2003. 